\title{
Measurement of the energy spectra of carbon and oxygen nuclei in cosmic rays with CALET
}

\author{
Paolo Maestro* for the CALET Collaboration ${ }^{\dagger}$ \\ Department of Physical Sciences, Earth and Environment \\ University of Siena, via Roma 56, 53100 Siena (Italy) \\ E-mail: paolo.maestro@pi.infn.it
}

\begin{abstract}
The Calorimetric Electron Telescope (CALET) was installed onboard the International Space Station in August 2015 and since October 2015 it is collecting data with smooth and continuous operations. The instrument consists of two layers of segmented plastic scintillators to identify the charge of individual elements from proton to iron, a thin imaging tungsten scintillating fiber calorimeter providing accurate particle tracking and complementary charge measurement, and a thick total absorption calorimeter made of lead-tungstate crystal logs. In addition to high precision measurements of the electron spectrum up to several TeV, CALET can measure the individual spectra and elemental composition of cosmic-ray nuclei from few tens of $\mathrm{GeV}$ to the PeV scale, which is of fundamental importance to shed light on the mechanism of acceleration and propagation of cosmic rays in the Galaxy. In this paper, preliminary measurements of the energy spectra of carbon and oxygen, based on the first three years of collected data, will be presented.
\end{abstract}

36th International Cosmic Ray Conference -ICRC2019-

July 24th - August 1st, 2019

Madison, WI, U.S.A.

\footnotetext{
${ }^{*}$ Speaker.

${ }^{\dagger}$ for collaboration list see PoS(ICRC2019)1177
} 


\section{Introduction}

Direct measurements of charged cosmic rays (CR) providing information on their elemental composition and energy spectra up to the PeV energy scale are fundamental to achieve a better understanding of their origin, mechanism of acceleration and propagation in the Galaxy. After recent observations of a hardening in the proton, helium $[1,2,3]$, and heavy nuclei spectra $[4,5]$ around a few hundred $\mathrm{GeV} / \mathrm{n}$, it has become extremely important to provide new precise measurements of the region of transition for each nuclear species and its extension to the TeV scale, in order to discriminate among several classes of theoretical models proposed to explain these unexpected features $[6,7,8,9,10]$.

The CALorimetric Electron Telescope (CALET) [11, 12], a space-based instrument optimized for the measurement of the all-electron spectrum $[13,14]$, recently reported results of the proton spectrum measured over the wide energy range from $50 \mathrm{GeV}$ to $10 \mathrm{TeV}$ for the first time with a single instrument in space [15]. Taking advantage of its wide dynamic range, large thickness and excellent charge identification capability, CALET is carrying out extensive measurements of individual chemical elements in $\mathrm{CR}$ up to iron in the energy range from few $\mathrm{GeV}$ up to $\sim 1 \mathrm{PeV}$. In this paper, we describe the analysis procedure for $\mathrm{C}$ and $\mathrm{O}$ flux measurements and present their preliminary energy spectra based on the data collected in 1124 days of CALET operation onboard the International Space Station (ISS). Results on proton, electron and heavy nuclei spectra are reported in other proceedings of this conference [16, 17, 18, 19, 20].

\section{CALET instrument}

CALET is an all-calorimetric instrument consisting of a pair of plastic scintillator hodoscopes used as CHarge Detector (CHD), a finely segmented pre-shower IMaging Calorimeter (IMC), and a Total AbSorption Calorimeter (TASC) for energy measurement. IMC is made of 7 tungsten plates interleaved with 8 double layers of 448 scintillating fibers ( $\mathrm{SciFi})$ each $\left(1 \mathrm{~mm}^{2}\right.$ cross-section, $448 \mathrm{~mm}$ length). SciFi are readout individually and arranged along orthogonal directions in each layer to reconstruct the CR particle direction. TASC is a homogeneous calorimeter made of Lead Tungstate (PWO) logs $\left(2 \times 1.9 \times 32 \mathrm{~cm}^{3}\right)$ arranged in 12 layers of 16 logs each. Each log is readout by a dual photodiode/avalanche-photodiode system, but in the first layer, where photomultipliers tube are used.

The total thickness of the CALET instrument is equivalent to $30 X_{0}$ and 1.3 proton interaction length $\left(\lambda_{I}\right)$. The geometrical factor is $0.12 \mathrm{~m}^{2} \mathrm{sr}$ and the total weight is $613 \mathrm{~kg}$.

CALET was launched on August 19, 2015 by the Japanese rocket H-II Transfer Vehicle (HTV-5) and installed on the Japanese Experiment Module Exposure Facility of the ISS for a 5-year mission ore more. The on-orbit commissioning phase aboard the ISS was successfully completed in the first days of October 2015, and since then the instrument has been taking science data continuously [21].

\section{Data analysis}

We have analyzed 1124 days of flight data (FD) collected from October 13, 2015 to October 31, 2018. Energy calibration of each channel of CHD, IMC and TASC is performed by using 
penetrating proton and He particles, selected in-flight by a dedicated trigger mode. Raw signals are corrected for non-uniformity in light output, gain differences among the channels, position and temperature dependence as well as temporal gain variations [22]. After calibration, each CR particle is reconstructed and each event assigned a charge and an energy. Carbon and oxygen events are then selected and sorted into energy intervals and the number of counts in each bin is corrected for several effects in order to compute the energy spectrum. Event display of a candidate carbon is shown in Fig. 2.

Detailed Monte Carlo (MC) simulations were developed, based respectively on FLUKA [23] and EPICS [24] packages and employing the hadronic interaction model DPMJET-III [25]; they are used to validate and tune the reconstruction method and evaluate event reconstruction efficiencies, background contaminations and the energy response matrix.

The steps of the analysis procedure are described in the following subsections.

\subsection{Preselection}

In this analysis, we have used events taken with the onboard high-energy (HE) trigger mode, designed to ensure maximum exposure to electrons above $10 \mathrm{GeV}$ and other high-energy shower events. An offline trigger confirmation is applied to HE-triggered events, by requiring that the signal in the top TASC layer (TASCX1) and the last two pairs of IMC SciFi layers are larger than the energy released respectively by 100 and 50 MIP ${ }^{1}$. HE trigger efficiency curves as a function of the TASC total energy deposit were measured using a subset of data taken with the same trigger logic, but lower thresholds (to detect also penetrating particles). Trigger curves for $\mathrm{C}$ and $\mathrm{O}$ turn out to be in good agreement with predictions from MC simulations, as shown in Fig. 2.

In order to remove possible events triggered by off-acceptance particles entering the TASC from
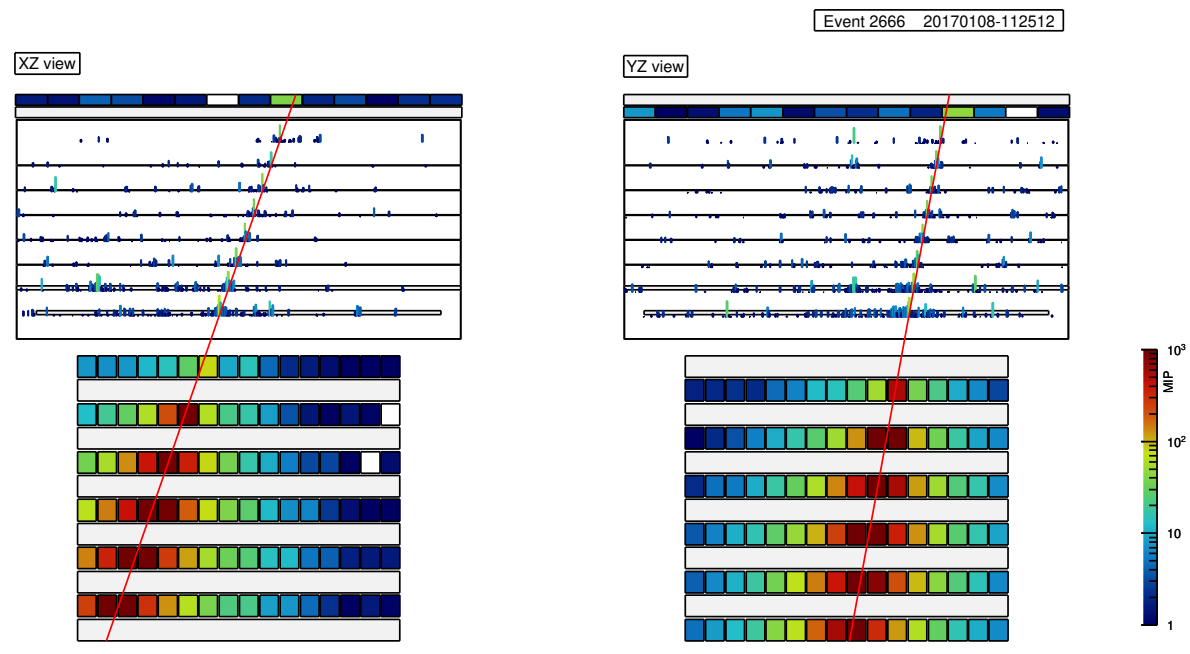

Figure 1: Image of a candidate carbon event with a shower energy in TASC of $\sim 11 \mathrm{TeV}$. The straight (red) lines represent the projections of the reconstructed particle trajectory in the $\mathrm{XZ}$ and $\mathrm{YZ}$ instrument views, respectively. The logarithmic color scale represents the signal amplitude (expressed in units of mip) detected in each CHD, IMC and TASC channel.

\footnotetext{
${ }^{1}$ minimum ionizing particle.
} 
lateral sides, the energy deposits in TASCX1 and in the lateral TASC crystals are required to be less than $40 \%$ of the total energy deposit in TASC.

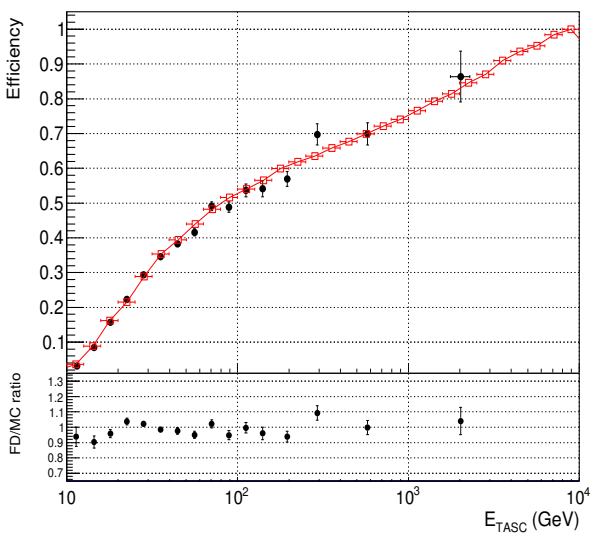

(a)

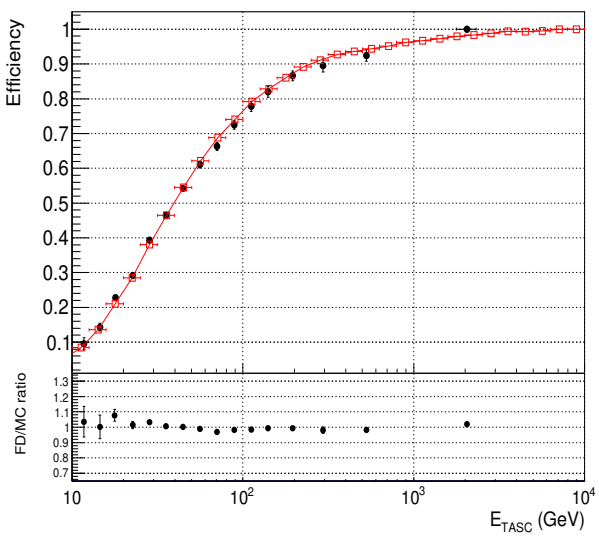

(b)

Figure 2: HE trigger efficiency as a function of the deposited energy in TASC for $\mathrm{C}(a)$ and $\mathrm{O}(b)$ nuclei as derived from flight FD (black dots) and MC (red rectangles). The FD to MC efficiency ratio is shown in the small canvas.

\subsection{Track reconstruction}

An original reconstruction method of the primary particle direction based on a combinatorial Kalman filter (KF) algorithm was developed [26]. This method exploits the fine granularity and imaging capability of the IMC and provides robust track finding and fitting, allowing to identify the incident CR track in a large amount of shower particle tracks backscattered from TASC. The track fitting algorithm has been extensively validated with simulated data and good agreement was found between MC and FD distributions of reconstructed incident angle and impact point coordinates on top of CHD. An angular resolution of $\sim 0.1^{\circ}$ is found for $\mathrm{C}$ and $\mathrm{O}$ nuclei. The resolution of the impact point on CHD is $\sim 220 \mu \mathrm{m}$.

Events with a well-fitted primary track crossing the detector from CHD top to TASC bottom, with $2 \mathrm{~cm}$ margins at TASCX1, are used in this analysis. Additional cuts on $\chi^{2} / \mathrm{ndf}(<10)$ of the track fit and the number of track points $(>4)$ in each view are applied to ensure good track quality.

\subsection{Charge identification}

The identification of the charge $Z$ of a CR particle is based on multiple measurements of its specific ionization $d E / d x$ in CHD paddles and IMC SciFi crossed by the reconstructed track. For each event, three independent $d E / d x$ measurements are obtained, one for each CHD layer and one by averaging the specific ionization yields in the first eight IMC layers. Corrections for light yield saturation in scintillator, due to high ionization density around the particle track, are applied based on fits of "halo" model functions [27, 28, 29] to CALET data (Fig. 3(a)). By using the fitted functions, three charge values are computed from the measured $d E / d x$ yields on an event-by-event basis.

The resulting distribution of the reconstructed charge combining the two CHD layers is shown in 


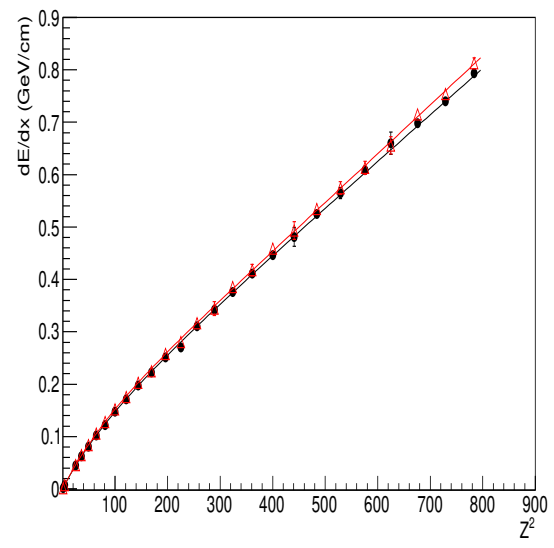

(a)

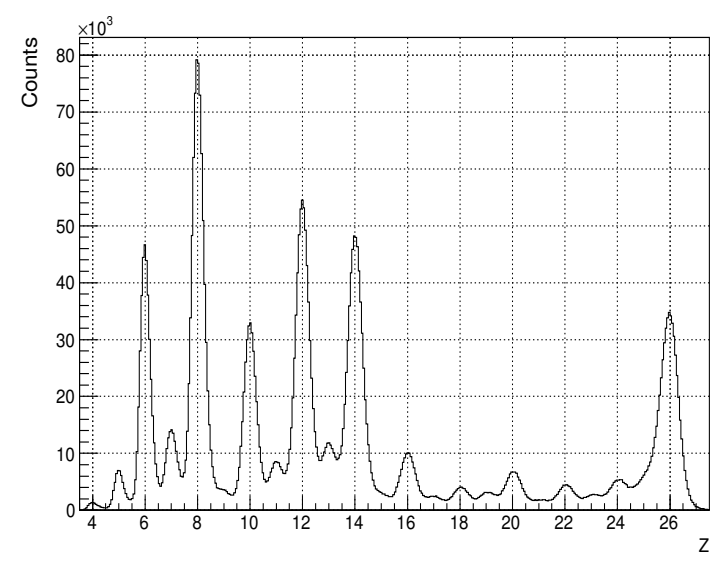

(b)

Figure 3: (a) Mean value of $d E / d x$ for top (black dots) and bottom (triangles) CHD layers as a function of nominal $Z^{2}$ of each nuclear species from $\mathrm{Be}$ to $\mathrm{Fe}$ in cosmic rays. The lines represent "halo" model functions $[28,29]$ fitted to the data. (b) Charge distribution in the elemental range from $\mathrm{Be}$ to $\mathrm{Fe}$, as measured by the combined $\mathrm{CHD}$ layers using a subset of FD.

Fig. 3(b). An excellent charge resolution $\sigma_{Z} \sim 0.15 e$ (charge unit) and $\sim 0.24 e$ is estimated for $\mathrm{CHD}$ and IMC, respectively, in the elemental range from $\mathrm{Be}$ to $\mathrm{O}$.

$\mathrm{C}$ and $\mathrm{O}$ candidates are identified by requiring the consistency of CHD and IMC charge measurements with nominal charge within $\pm 2 \sigma_{Z}$. Residual background due to charge-changing nuclear interactions occurring in the upper part of the instrument are removed by requiring the consistency, within $30 \%$, between the mean $d E / d x$ values measured with the first 4 IMC layers in each view.

\subsection{Energy measurement}

The shower energy of each event is calculated as the sum of the calibrated energy deposits $\left(E_{T A S C}\right)$ of all the TASC channels. Distributions of $E_{\text {TASC }}$ for $\mathrm{C}$ and $\mathrm{O}$ candidate events selected in this analysis are shown in Fig. 4, together with the background expected from other nuclei misidentified as $\mathrm{C}$ or $\mathrm{O}$. It can be noticed that the total background in the selected $\mathrm{C}$ and $\mathrm{O}$ event samples is negligible: $<1 \%$ up to $E_{T A S C} \sim 5 \times 10^{3} \mathrm{GeV}$, and $\sim 2 \%$ above.

Unlike for electrons, the energy released in TASC by interacting CR nuclei is only a fraction of the primary particle energy with large event-to-event fluctuations, because of the incomplete longitudinal containment of the hadronic showers in a $1.3 \lambda_{I}$ thick calorimeter. For flux measurement, energy unfolding is applied to correct $E_{T A S C}$ distributions of selected $\mathrm{C}$ and $\mathrm{O}$ candidates for significant bin-to-bin migration effects (due to the limited energy resolution) and infer the primary particle energy. In this analysis, we apply the iterative unfolding method based on the Bayes' theorem [30] implemented in the RooUnfold package [31]. The response matrix (each element of which represents the probability that primary nuclei in a certain energy interval of the CR spectrum produce an energy deposit in a given $E_{T A S C}$ bin) is derived using $\mathrm{MC}$ simulation after applying the same selection procedure as for FD and used in the unfolding procedure.

The simulated energy response was validated and tuned with beam test data, taken as CERN SPS in 2015 using a beam of accelerated ion fragments with $A / Z=2$ and kinetic energy of $150 \mathrm{GeV} / \mathrm{n}$ 


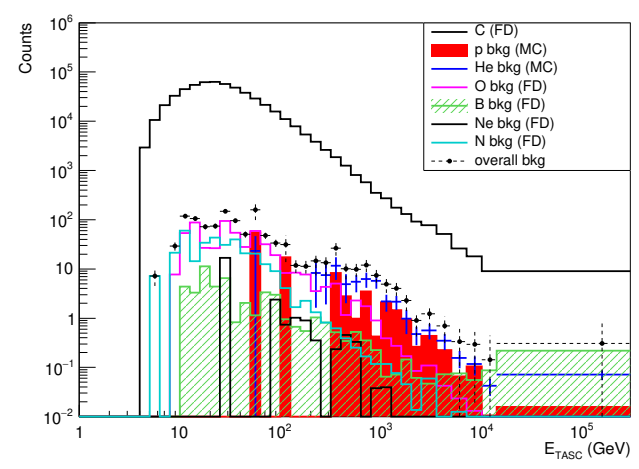

(a)

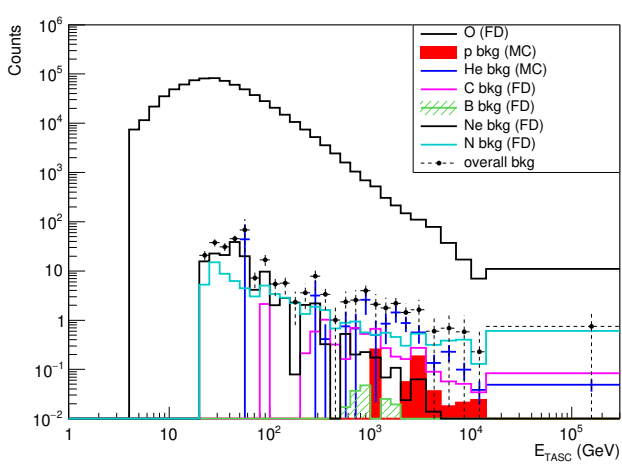

(b)

Figure 4: Distribution of the total energy deposited in TASC by the selected $\mathrm{C}(a)$ and $\mathrm{O}(b)$ nuclei in FD (black line) and contamination from different nuclei. Overall background is represented by the black dots.

[32]. The mean energy deposited in TASC by different nuclear species in the beam (selected with CHD) is plotted as a function of the kinetic energy per particle in Fig. 5(a). The energy response of TASC is linear up to the maximum available particle energy of $\sim 6 \mathrm{TeV}$ (obtained with a primary beam of ${ }^{40} \mathrm{Ar}$ nuclei); the fraction of particle energy released in the TASC is $\sim 20 \%$. The energy resolution is $\sim 30 \%$ (Fig. 5(b)), nearly independent on energy.

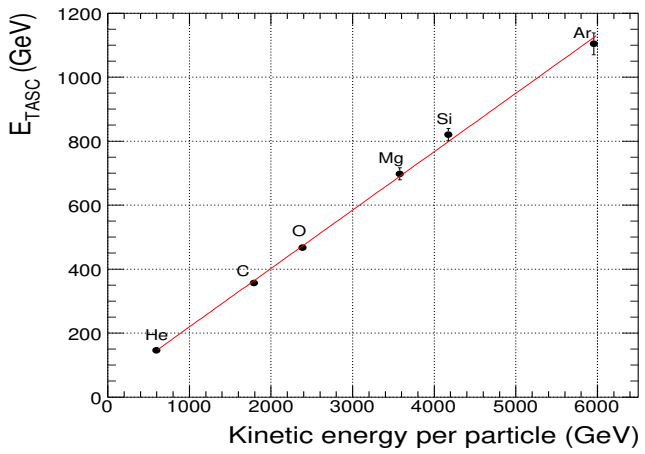

(a)

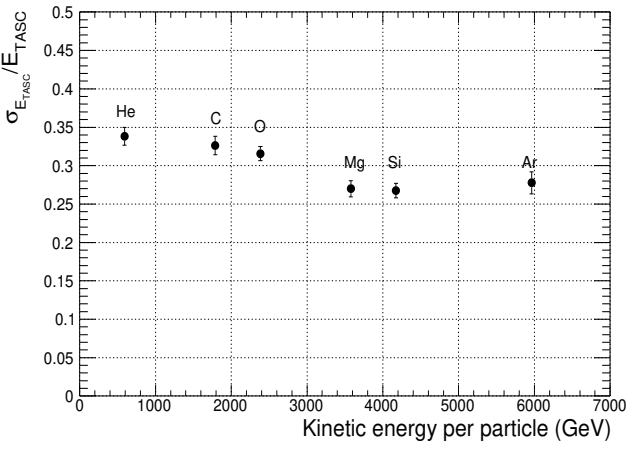

(b)

Figure 5: Energy linearity $(a)$ and resolution $(b)$ of TASC as measured at CERN SPS with beams of $150 \mathrm{GeV} / \mathrm{n}$ ion fragments with $A / Z=2$.

\section{Preliminary energy spectrum}

The energy spectrum is calculated as

$$
\begin{gathered}
\Phi(\hat{E})=\frac{N(E)}{\Delta E \times \varepsilon(E) \times S \Omega \times T} \\
N(E)=U\left(N_{o b s}\left(E_{T A S C}\right)-N_{b g}\left(E_{T A S C}\right)\right)
\end{gathered}
$$

where $\hat{E}$ is the median kinetic energy of the $[E, E+\Delta E)$ bin, $\Delta E$ the energy bin width, $\varepsilon(E)$ the overall selection efficiency, $T$ is the exposure time ( $\sim 84 \%$ of total observation time), $S \Omega$ the 
"fiducial" geometrical acceptance $\left(\sim 510 \mathrm{~cm}^{2} \mathrm{sr}\right), N(E)$ the bin content in the unfolded distribution, $U()$ is the unfolding procedure based on Bayes' theorem, $N_{o b s}\left(E_{T A S C}\right)$ the bin content of $E_{T A S C}$ distribution (including background), $N_{b g}\left(E_{T A S C}\right)$ the bin content of background events in $E_{T A S C}$ distribution.

The preliminary energy spectra of CR carbon and oxygen nuclei measured with CALET in 1124 days of operation are shown in Fig. 6. CALET measurements, covering an interval of kinetic energy per particle from 150 to $\sim 10^{5} \mathrm{GeV}$, are compared with previous observations from spacebased $[5,33,34,35]$ and balloon-borne $[36,37,38,39,40]$ experiments.

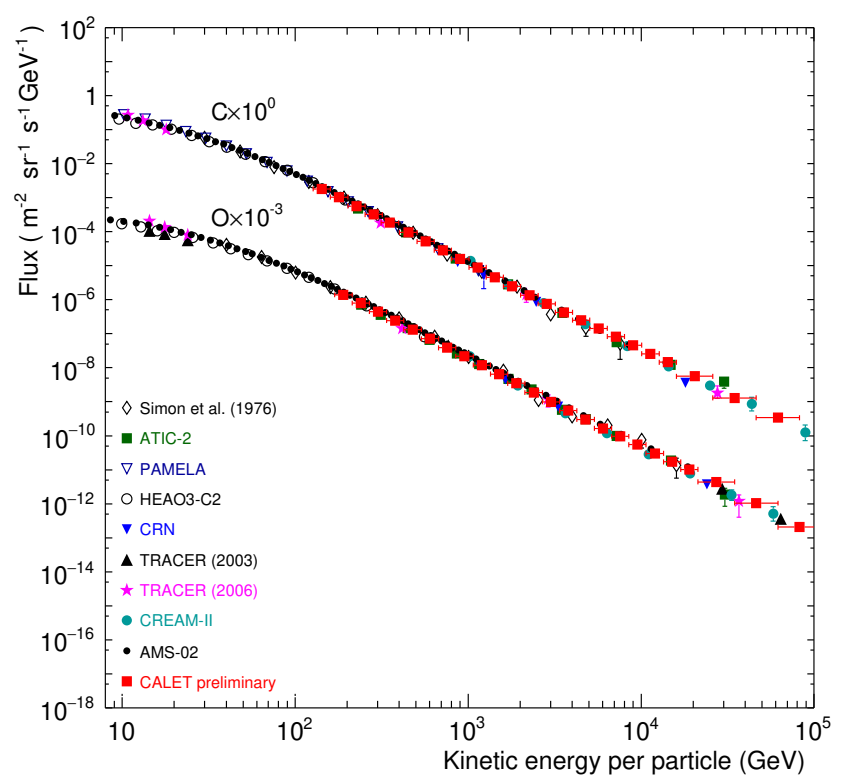

Figure 6: Preliminary fluxes of CR carbon and oxygen nuclei measured with CALET (red markers) as a function of kinetic energy per particle, compared with previous direct observations [5, 33, 34, 35, 36, 37, 38, 39, 40]. Error bars of CALET data represent the statistical uncertainty only.

\section{Summary}

The preliminary results of carbon and oxygen spectra demonstrate the excellent capability of CALET to measure heavy nuclei in CR with high statistics, excellent charge separation and over a wide energy range. Further analysis with an increased data set and including a detailed assessment of systematic uncertainties, is ongoing, aimed at investigating possible spectral features in the CR spectra.

\section{References}

[1] M. Aguilar et al., Phys. Rev. Lett. 114 (2015) 171103; Phys. Rev. Lett. 115 (2015) 211101

[2] O. Adriani et al., Science 332 (2011) 69-72

[3] Y. S. Yoon et al., Astrophys. J. 728 (2011) 122; Astrophys. J. 839 (2017) 5 
[4] H. S. Ahn et al., Astrophys. J. Lett. 714 (2010) L89

[5] M. Aguilar et al., Phys. Rev. Lett. 119 (2017) 251101

[6] P. Serpico, Proc. of Science (ICRC2015) 009

[7] S. Thoudam \& J. R. Hörandel, Astron. Astrophys. 567 (2014) A33

[8] N. Tomassetti, Astrophys. J. Lett. 752 (2012) L13

[9] A. Vladimirov, G. Johannesson, I. Moskalenko \& T. Porter, Astrophys. J. 752 (2012) 68

[10] Y. Ohira, N. Kawanaka \& K. Ioka, Phys. Rev. D 93 (2016) 083001

[11] S. Torii \& P.S. Marrocchesi, Adv. in Sp. Res. (2019) in press

[12] S. Torii, Proc. of Science (ICRC2017) 1092

[13] O. Adriani et al., Phys. Rev. Lett. 119 (2017) 181101

[14] O. Adriani et al., Phys. Rev. Lett. 120 (2018) 261102

[15] O. Adriani et al., Phys. Rev. Lett. 122 (2019) 181102

[16] P.S. Marrocchesi, Proc. of Science (ICRC2019) 531

[17] Y. Akaike, Proc. of Science (ICRC2019) 386

[18] Y. Asaoka, Proc. of Science (ICRC2019) 678

[19] S. Torii, Proc. of Science (ICRC2019) 182

[20] B. Rauch, Proc. of Science (ICRC2019) 119

[21] Y. Asaoka et al., Astroparticle Physics 100 (2018) 29

[22] Y. Asaoka et al., Astroparticle Physics 91 (2017) 1

[23] T. T. Böhlen et al., Nuclear Data Sheets 120 (2014) 211

[24] K. Kasahara, Proc. of $24^{\text {th }}$ ICRC, Vol. 1 (1995) 399

[25] S. Roesler, R. Engel \& J. Ranft, Proc. of the Monte Carlo Conference, Lisbon (2000) 1033

[26] P. Maestro \& N. Mori, Proc. of Science (ICRC2017) 208

[27] P. S. Marrocchesi et al., Nucl. Instr. and Meth. A 659 (2011) 477

[28] R. Voltz, J. Lopes da Silva, G. Laustriat \& A. Coche, JChPh 45 (1966) 3306

[29] G. Tarlé et al., Astrophys. J. 230 (1979) 607

[30] G. D'Agostini, Nucl. Instr. and Meth. A 362 (1995) 487

[31] T. Adye, arXiv:1105.1160v1 (2011)

[32] Y. Akaike, Proc. of Science (ICRC2015) 613

[33] J. J. Engelmann et al., Astron. Astrophys. 233 (1990) 96

[34] D. Müller et al., Astrophys. J. 374 (1991) 356

[35] O. Adriani et al., Astrophys. J. 791 (2014) 93

[36] M. Simon et al., Astrophys. J. 239 (1980) 712

[37] A. D. Panov et al., Bull. Russian Acad. Sci. 73 (2009) 564

[38] M. Ave et al., Astrophys. J. 678 (2008) 262

[39] A. Obermeier et al. Astrophys. J. 742 (2011) 14

[40] H. S. Ahn et al., Astrophys. J. 707 (2009) 593 